\title{
Early results of AGN studies with the space VLB interferometer RadioAstron
}

\author{
Yuri Kovalev \\ Astro Space Center of Lebedev Physical Institute, Russia \\ Email: yyk@asc.rssi.ru
}

\begin{abstract}
The space element of the ground-space very long baseline (VLB) interferometer RadioAstron is a 10-meter radio telescope Spektr-R which was successfully launched in 2011. It covers four frequency bands from 0.3 to $25 \mathrm{GHz}$ and provides baselines up to $350,000 \mathrm{~km}$. This allows to study space objects with a resolution as high as about 10 microarcseconds. Fringes are found at all four bands of 92, 18,6, and $1.3 \mathrm{~cm}$. Science observations are ongoing. Early results of the RadioAstron AGN survey at extreme angular resolutions will be presented in the talk. In particular, AGN core emission is successfully detected at interferometer baselines up to 7.6 GLambda (or fringe spacing 27 microarcseconds) which is the current VLBI record. High radio brightness of AGN cores is found significantly above the known inverse-Compton limit. Implications to AGN jet emission models will be discussed. First results of RadioAstron space VLBI imaging of AGN jets will be also presented.
\end{abstract}

